\title{
Análise de imagens no estudo morfológico e fisiológico de sementes de abóbora
}

\author{
Patricia P Silva $^{1}$; Raquel A Freitas ${ }^{1}$; Silvio M Cícero²; Júlio Marcos-Filho²; Warley M Nascimento ${ }^{1}$ \\ ${ }^{1}$ Embrapa Hortaliças, C. Postal 218, 70351-970 Brasília-DF; warley.nascimento@embrapa.br; ${ }^{2}$ ESALQ-Depto. Prod. Vegetal, C. Postal \\ 9, 13418-900 Piracicaba-SP
}

\section{RESUMO}

A obtenção de sementes de abóbora com alta qualidade fisiológica é fundamental para colocar a produção nacional em condições de competitividade com as sementes importadas. Uma técnica que vem sendo utilizada recentemente para estudos relacionados à elucidação de vários aspectos do comportamento das sementes é a análise de imagens, incluindo as obtidas por meio do raio X. Esta técnica consiste em radiografar sementes com o objetivo de avaliar a morfologia interna e, assim, estabelecer relações com o desempenho da semente. $\mathrm{O}$ objetivo do presente estudo foi verificar se a técnica de raio $\mathrm{X}$ pode ser utilizada com sucesso na detecção de anormalidades e seus reflexos na qualidade fisiológica de sementes de abóbora. Foram utilizadas sementes de abóbora 'Brasileirinha' provenientes de frutos com diferentes estádios de maturação $(30,40,50$ e 60 dias após a antese). Em cada época, as sementes foram extraídas imediatamente após a colheita ou após o armazenamento dos frutos por 15 dias. No teste de raio $\mathrm{X}$ as sementes foram submetidas à intensidade de $25 \mathrm{kV}$ por 40 segundos e, em seguida, foi conduzido teste de germinação sob temperatura alternada de $20-30^{\circ} \mathrm{C}$. Após quatro dias foi realizada a primeira contagem e após oito dias a última leitura, computando-se as porcentagens de plântulas normais, anormais e as sementes mortas. Após a obtenção do resultado do teste de germinação foi feita comparação desses resultados com imagens da morfologia interna de cada semente, obtidas por meio do raio X. Foi constatado que a técnica de raio $\mathrm{X}$ é eficiente na detecção de sementes mal formadas bem como de sementes vazias de abóbora e estas afetam negativamente sua germinação.
\end{abstract}

\begin{abstract}
Image analysis to associate morphological and physiological characteristics in pumpkin seeds

Obtain pumpkin seeds with high physiological quality is critical for the domestic seed production in terms of competitiveness with imported seeds. One technique that has recently been used to elucidate the various aspects of seed performance is the analysis of images, including those obtained through X-ray. This technique involves X-ray images of seeds to evaluate the internal morphology and to establish the relationship with the seed performance. Thus, the objective of this study was to determine whether the X-ray technique may help to detect internal abnormalities and to assess the quality of pumpkin seeds. 'Brasileirinha' pumpkin fruits were harvested at different maturation stages (30, 40, 50 and 60 days after anthesis). In each stage, seeds were extracted either immediately after harvesting or after 15 days of fruit storage. In the X-ray test, seeds were subjected to an intensity of $25 \mathrm{kV}$ for 40 seconds and then a germination test was carried out at alternate temperatures $\left(20-30^{\circ} \mathrm{C}\right)$. The first count was held after four days and the last reading after eight days, computing the percentages of normal and abnormal seedlings and dead seeds. After obtaining the results of the germination test, a comparison of results was done with the images of the internal morphology of each seed, obtained by means of X-ray. The use of X-ray technique is effective in detecting malformed and empty pumpkin seeds and these negatively affect germination.
\end{abstract}

Palavras-chave: Cucurbita moschata, maturação, teste de raio-X, germinação.

Keywords: Cucurbita moschata, maturation, X-rays, germination.

(Recebido para publicação em 19 de março de 2013; aceito em 25 de março de 2014) (Received on March 19, 2013; accepted on March 25, 2014)

\begin{abstract}
A s abóboras, do ponto de vista sócio econômico, são importantes por fazerem parte da alimentação básica das populações de várias regiões do Brasil, tendo alcançado em 2008 o volume comercializado de 90.606 toneladas (Talamini \& Ramos, 2011). Apenas no segmento de abóbora japonesa (tipo Tetsukabuto), o mercado de sementes foi estimado, em 2007, em cerca de 14 t de sementes totalizando $\mathrm{R} \$ 10$ milhões (ABCSem, 2007). Parte da quantidade de sementes de abóbora utilizadas no País é importada. Nesse aspecto, a solução para problemas relacionados à obtenção de sementes de elevada qualidade fisiológica é fundamental
\end{abstract}

para colocar a produção nacional em condições de competitividade com as sementes importadas. As informações disponíveis sobre o assunto são escassas e inconclusivas em relação a aspectos importantes, como a melhor época para a realização da colheita dos frutos, o período necessário para que as sementes atinjam a maturidade fisiológica e procedimentos adequados para a avaliação da qualidade fisiológica das sementes.

Sabe-se que a partir da maturidade fisiológica, a deterioração é contínua e irreversível; assim, a determinação do ponto de maturidade fisiológica da semente assume grande importância na racionalização da colheita que objetive a produção de sementes de elevada qualidade fisiológica e, até certo ponto, qualidade sanitária. Sabe-se que, quanto maior o tempo decorrido entre o ponto de maturidade fisiológica e a colheita, mais sujeita está a semente às adversidades climáticas e ao ataque de insetos e microrganismos patogênicos. $\mathrm{O}$ momento da maturidade fisiológica das sementes varia acentuadamente em função das condições ambientais predominantes, da espécie ou cultivar utilizados, podendo, em alguns casos, ser associado a modificações nas características morfológicas dos frutos e sementes, o que facilita a sua identificação no campo. Em outros casos, principalmente quando se trata de 
frutos carnosos, como é o caso da abóbora, o ponto de maturidade fisiológica pode ser influenciado por um período de repouso pós-colheita dos frutos, antes da extração das sementes. Nestes casos, há relatos de que sementes provenientes de frutos imaturos podem apresentar qualidade fisiológica comparável à das sementes extraídas de frutos maduros, desde que estes frutos imaturos sejam convenientemente armazenados antes da extração das sementes (Pedrosa et al., 1987; Barbedo et al., 1994; Nascimento et al., 2000, respectivamente para abóbora, pepino e berinjela).

O contínuo progresso do conhecimento sobre análise de sementes envolve estudos nas áreas de fisiologia, bioquímica, biologia molecular e biofísica. A análise de imagens também representa uma ferramenta relativamente recente, com potencial indiscutível para estudos básicos e aplicados, visando à elucidação de vários aspectos do comportamento das sementes e o aprimoramento da metodologia para a avaliação de diferentes atributos da qualidade de sementes (Carvalho \& Oliveira, 2006).

Os recursos utilizados para a análise de imagens digitais, geralmente associados à informática, consistem fundamentalmente da captura de imagens de sementes, de plântulas ou de suas partes constituintes, por meio de câmera fotográfica, filmadora ou "scanner", com objetivos de identificação, verificação de sua integridade ou o estabelecimento de relações com o desempenho da semente. Esses procedimentos permitem apenas a avaliação de imagens externas das sementes, de modo que sua combinação com análises utilizando raio $\mathrm{X}$ permite ampliar a diagnose. Os raios X não causam danos às sementes, sendo possível utilizá-las para a condução de testes fisiológicos, com o objetivo de confrontar as observações da morfologia interna com as plântulas (normais ou anormais) ou sementes mortas resultantes dos testes fisiológicos. Além disso, as imagens obtidas poderão ser catalogadas, arquivadas e utilizadas posteriormente para a definição de critérios de avaliação não subjetivos, padronizados e, consequentemente, mais precisos que os provenientes dos baseados apenas na visão humana (Menezes et al., 2005;
ISTA, 2004).

A situação atual do conhecimento sobre o uso potencial da análise de imagens em diversos segmentos da avaliação da qualidade de sementes ainda é muito restrita diante da ampla diversidade de espécies produzidas, sistemas de produção e de problemas que exigem procedimentos mais eficientes para solução. Assim, este trabalho teve como objetivo básico focalizar o tema análise de imagens de raio $\mathrm{X}$ no estudo sobre morfologia interna e monitoramento da formação de sementes. Independentemente do fator de variação, a meta fundamental foi verificar até que ponto e em que situações essas técnicas de análise digitais processadas em computador viabilizam o estabelecimento de diagnósticos associados ao desempenho de amostras de sementes. Utilizou-se para isso, a espécie abóbora (Cucurbita moschata) como modelo. As informações obtidas neste estudo poderão ser estendidas ou utilizadas para outras espécies de cucurbitáceas ou mesmo para outras olerícolas.

\section{MATERIAL E MÉTODOS}

Estudo da maturação das sementes - Sementes de abóbora 'Brasileirinha' foram produzidas na Embrapa Hortaliças, Brasília-DF. Por ocasião do florescimento, flores em antese foram selecionadas e etiquetadas, ao acaso. As colheitas dos frutos foram realizadas aos 30, 40, 50 e 60 dias após a antese (DAA). Em cada época, os frutos, após a colheita, foram armazenados em laboratório a $25^{\circ} \mathrm{C}$, por zero (extração das sementes imediatamente à colheita) ou por 15 dias. As sementes foram extraídas dos frutos e a mucilagem foi removida utilizando cal hidratada. Em seguida, as sementes foram lavadas em água corrente, secadas à temperatura ambiente por $24 \mathrm{~h}$ e, posteriormente, transferidas para uma câmara com ventilação, a $32^{\circ} \mathrm{C}$ por mais $24 \mathrm{~h}$. Após a secagem, as sementes foram beneficiadas em soprador pneumático e realizada a determinação do grau de umidade, de acordo com as Regras para Análise de Sementes (Brasil, 2009).

Utilização de raio X e digitalização de imagens na avaliação da morfologia interna das sementes - Os testes foram realizados no Laboratório de Análises de Imagens e no Laboratório de Análise de Sementes da ESALQ em Piracicaba-SP. O teste de raio $\mathrm{X}$ foi realizado com quatro repetições de 25 sementes de cada tratamento (frutos colhidos aos 30, 40, 50 e 60 DAA). As sementes foram colocadas em alvéolos de uma placa de acrílico e numeradas de acordo com a posição ocupada, de maneira que pudessem ser identificadas nas determinações posteriores. A placa de acrílico contendo as sementes foi colocada diretamente sobre um filme de raio X (Kodak MIN-R EV 2000, tamanho $18 \times 24 \mathrm{~cm})$. As imagens foram obtidas com intensidade de $25 \mathrm{kV}$ e 40 segundos de exposição, utilizando-se equipamento FAXITRON X-Ray, modelo MX-20. A revelação foi efetuada em uma processadora Hope X-Ray, modelo 319 Micromax. As imagens dos filmes de raio $\mathrm{X}$ foram capturadas por um Scanner Umax, modelo Power Look 1100, para ampliação e visualização em computador.

Após a revelação das radiografias, as sementes foram classificadas de acordo com sua morfologia interna em a) sementes cheias [aquelas com áreas vitais (tecidos essenciais) normalmente estruturadas]; b) sementes mal formadas; c) sementes vazias.

Teste de germinação para avaliação de características e desempenho de sementes de abóbora - Foi realizado o teste de germinação com as sementes previamente radiografadas e identificadas, determinando-se a porcentagem inicial de germinação (primeira contagem) e final (última contagem). As sementes foram colocadas em grupo de 25 , sendo cinco fileiras de cinco sementes distribuídas sobre duas folhas de papel germitest, cobertas com mais uma folha e enroladas. As folhas de papel foram previamente umedecidas com quantidade de água equivalente a 2,5 vezes a massa do substrato (papel). Os rolos foram mantidos em germinadores tipo BOD em temperatura alternada de 20-30 ${ }^{\circ} \mathrm{C}$. Após quatro dias, foi realizada a primeira contagem e após oito dias, a contagem final, onde foram computadas as plântulas normais, anormais e 
as sementes mortas. Após a obtenção do resultado de germinação, foi feita a comparação desse resultado com as imagens de raio $X$.

$\mathrm{O}$ experimento foi conduzido no delineamento inteiramente casualizado, com quatro repetições. Os dados foram submetidos à análise de variância, sendo a comparação de médias efetuada pelo teste de Tukey, a 5\% de probabilidade.

\section{RESULTADOS E DISCUSSÃO}

As sementes apresentaram teor de água de 6,5 a 7,2\%. O teor de água é um fator de grande importância no estudo de raio $X$, pois irá refletir diretamente na qualidade da imagem. Assim, a padronização do teor de água das sementes antes da realização do teste de raio $\mathrm{X}$ é uma prática que deve ser enfatizada. Por exemplo, o mínimo de $12 \%$ de umidade das sementes foi indicado para uma maior visualização das imagens obtidas pelo raio X em pimentão (Gagliardi \& Marcos Filho, 2011).

A regulagem do aparelho de raio $X$ depende da espessura, densidade e composição da semente e do aparelho utilizado (Bino et al., 1993; ISTA, 2004). $\mathrm{O}$ período de exposição das sementes à radiação de 40 segundos e à intensidade de $25 \mathrm{kV}$ possibilitaram uma visualização adequada das sementes de abóbora nas radiografias; nestas condições, foi

Tabela 1. Número de sementes cheias (SC), mal formadas (SM) ou vazias (SV) de abóbora (Cucurbita moschata) 'Brasileirinha' provenientes de frutos colhidos em diferentes estádios de maturação (DAA) e extraídas em duas épocas [number of full seeds (SC), damaged (SM) or empty seeds (SV) of 'Brasileirinha' pumpkin (Cucurbita moschata), at different periods of maturation and extraction]. Brasília, Embrapa Hortaliças, 2011.

\begin{tabular}{lcccc}
\hline Frutos (DAA) & $\begin{array}{c}\text { Extração } \\
\text { (dias após a colheita) }\end{array}$ & SC (\%) & SM (\%) & SV (\%) \\
\hline 30 & 0 & 0 & 0 & 100 \\
\hdashline & 15 & 0 & 0 & 100 \\
\hline & 0 & 0 & 5 & 45 \\
\hline & 15 & 55 & 39 & 6 \\
\hline & 0 & 25 & 71 & 4 \\
& 15 & 54 & 45 & 1 \\
\hline & 0 & 38 & 60 & 2 \\
\hline & 15 & 62 & 38 & 0 \\
\hline
\end{tabular}

Tabela 2. Primeira contagem de germinação (PC) e germinação $(\mathrm{G})$ de sementes de abóbora (Cucurbita moschata) 'Brasileirinha' provenientes de frutos colhidos em diferentes estádios de maturação (DAA) e extraídas em duas épocas. ['Brasileirinha' pumpkin (Cucurbita moschata) first count (PC) and germination $(\mathrm{G})$ of seeds from fruits at different periods of maturation and extraction]. Brasília, Embrapa Hortaliças, 2011.

\begin{tabular}{|c|c|c|c|}
\hline Frutos (DAA) & $\begin{array}{c}\text { Extração } \\
\text { (dias após a colheita) }\end{array}$ & PC (\%) & $G(\%)$ \\
\hline \multirow{2}{*}{30} & 0 & $0 \mathrm{c}$ & $0 \mathrm{c}$ \\
\hline & 15 & $0 \mathrm{c}$ & $0 \mathrm{c}$ \\
\hline \multirow{2}{*}{40} & 0 & $0 \mathrm{c}$ & $0 \mathrm{c}$ \\
\hline & 15 & $0 \mathrm{c}$ & $55 \mathrm{~b}$ \\
\hline \multirow{2}{*}{50} & 0 & $0 \mathrm{c}$ & $3 \mathrm{~d}$ \\
\hline & 15 & $18 b$ & $54 \mathrm{~b}$ \\
\hline \multirow{2}{*}{60} & 0 & $0 \mathrm{c}$ & $13 \mathrm{~cd}$ \\
\hline & 15 & $27 \mathrm{a}$ & $65 \mathrm{ab}$ \\
\hline $\mathrm{CV}(\%)$ & & 20,25 & 9,12 \\
\hline
\end{tabular}

Médias seguidas pela mesma letra em cada coluna, não diferem entre si pelo teste de Tukey, a $5 \%$ de probabilidade (means followed by the same letter in each column do not differ by Tukey test at $5 \%$ probability). possível visualizar o desenvolvimento da estrutura das sementes de abóbora. A tonalidade observada na análise de imagens radiográficas é definida em função do nível de absorção da radiação em regiões distintas da semente, que é determinada pela espessura, densidade e composição dos tecidos da semente (Simak, 1991; ISTA, 1993). Com isso, sementes que não possuem tecido embrionário, por não apresentarem resistência à passagem dos raios $\mathrm{X}$, fornecem imagens escuras.

Observou-se que somente os tratamentos representados por sementes provenientes de frutos colhidos aos 60 DAA e armazenados por 15 dias não apresentaram sementes vazias. Com relação às sementes classificadas como mal formadas, somente aquelas oriundas de frutos colhidos aos 30 DAA não apresentaram esse tipo de semente, uma vez que todas essas sementes foram classificadas como vazias (Tabela 1 ). As sementes cheias foram observadas em vários tratamentos. Como esperado, quanto maior o tempo de maturação dos frutos, maior foi o número de sementes cheias.

A técnica de raio $X$ auxilia na avaliação da viabilidade das sementes (Swaminathan \& Kamra, 1961; Oliveira et al., 2003). Para se obter um resultado confiável é preciso estabelecer uma relação entre as estruturas internas das sementes, observadas na radiografia, com as respectivas plântulas ou sementes mortas. De um modo geral, observou-se pelo teste de germinação que os tratamentos com maior porcentagem de sementes cheias proporcionaram um resultado superior em relação aos outros tratamentos (Tabela 2). Resultados semelhantes foram obtidos por Carvalho et al. (2009) em sementes de abóbora, onde foi observado que os lotes com maior número de sementes cheias, apresentaram maior porcentagem de germinação e que as sementes danificadas fisicamente interferiram negativamente na germinação.

Verificou-se, ainda, que os resultados obtidos no teste de germinação foram concordantes com a classificação de sementes no teste de raio $\mathrm{X}$, sendo constatada, no entanto, a presença de plântulas normais e anormais nas 


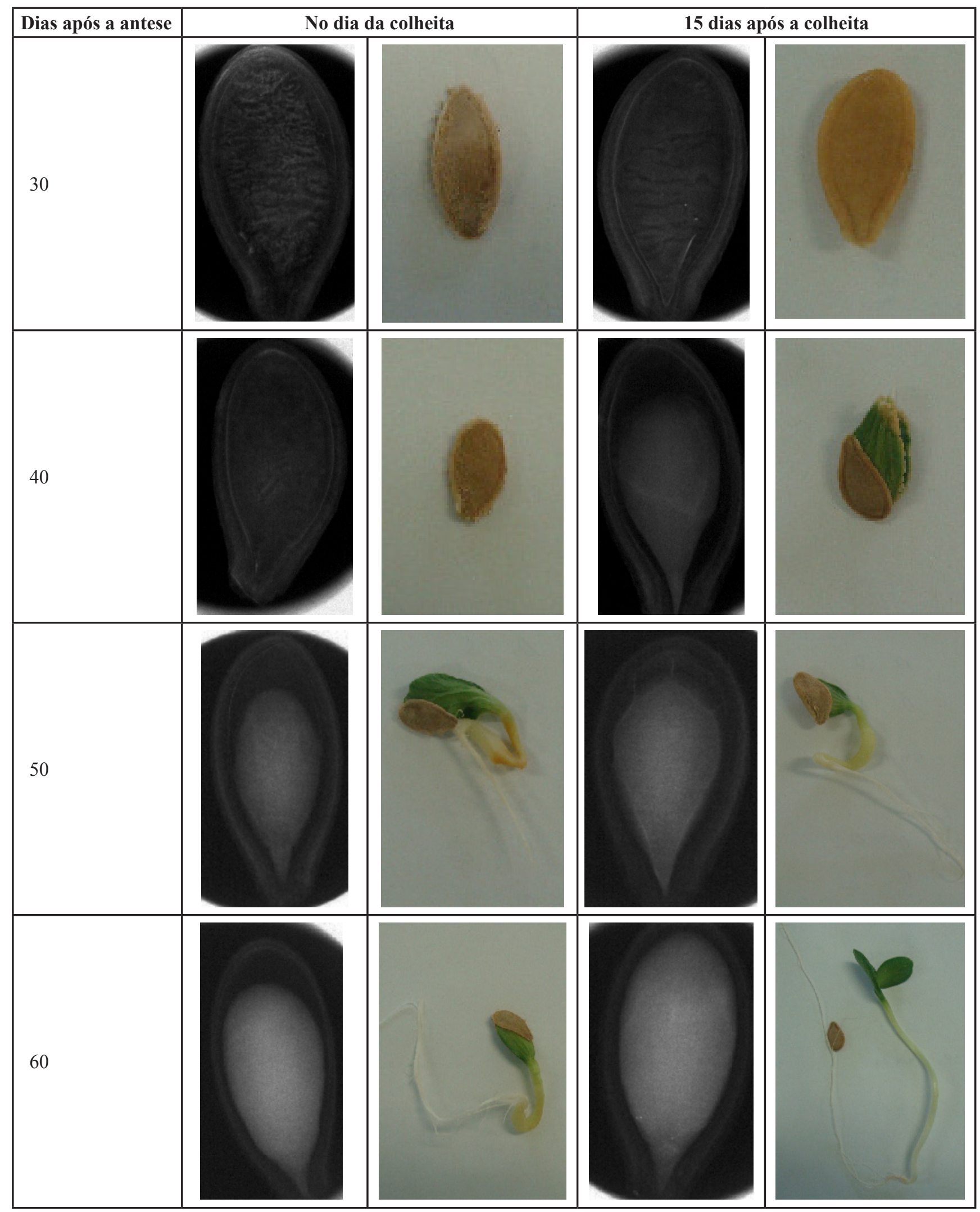

Figura 1. Imagens radiográficas de sementes de abóbora (Cucurbita moschata) 'Brasileirinha' e suas respectivas plântulas no final do período de germinação. Sementes não germinadas de frutos com 30 DAA (0 e 15 dias de armazenamento); plântulas anormais de frutos com 40 DAA (0 e 15 dias de armazenamento) e 50 DAA (0 e 15 dias de armazenamento); e plântulas normais de frutos com 60 DAA (0 e 15 dias de armazenamento) [X-ray images from 'Brasileirinha' squash (Cucurbita moschata) seeds and seedlings at the end of germination period. Non germinated seeds from fruits at 30 DAA ( 0 and 15 days after storing); abnormal seedlings from fruits of 40 DAA (0 and 15 days after storing) and 50 DAA ( 0 and 15 days after storing); and normal seedlings from 60 DAA (0 and 15 days after storing)]. Brasília, Embrapa Hortaliças, 2011. 
sementes classificadas como cheias (Figura 1). É importante destacar que no teste de raio $\mathrm{X}$ é possível visualizar, detalhadamente, a morfologia interna da semente, ou seja, se a semente está bem formada ou se apresenta problemas, como injúrias mecânicas, causadas por insetos, presença de tecidos em estado avançado de deterioração, entre outros. Entretanto, algumas sementes bem formadas e aparentemente sem nenhum problema podem dar origem a plântulas anormais ou sementes mortas, pois o teste de raio $\mathrm{X}$ não consegue detectar determinados problemas fisiológicos, como, por exemplo, fases intermediárias do processo de deterioração, que poderiam estar afetando o processo de germinação (Burg et al., 1994).

A maior parte das sementes consideradas cheias originou plântulas normais e a maioria das classificadas como mal formadas originou plântulas anormais. Por outro lado, $100 \%$ das sementes consideradas vazias não germinaram. Resultados semelhantes foram obtidos por Pupim et al. (2008) em sementes de embaúba. Carvalho et al. (2009), analisando sementes de abóbora também observaram que os lotes que possuíam maior número de sementes cheias originaram maior número de plântulas normais.

Os resultados obtidos no presente estudo confirmam as afirmações de que a morfologia interna das sementes é um indicativo do seu potencial de viabilidade. Dessa forma, a identificação e a posterior eliminação de sementes que apresentam má formação durante a produção e, ou problemas durante o processo de extração e beneficiamento, passíveis de serem visualizados nas radiografias, poderiam promover a melhoria da qualidade física e fisiológica de lotes de sementes. A utilização da técnica de raio $\mathrm{X}$ mostrou-se eficiente na detecção de sementes mal formadas e de sementes vazias de abóbora que afetam negativamente sua germinação.

\section{AGRADECIMENTOS}

À Fundação de Amparo à Pesquisa do Estado de São Paulo (FAPESP) pelo suporte financeiro para a realização da pesquisa.

\section{REFERÊNCIAS}

ABCSem - ASSOCIAÇÃO BRASILEIRA DO COMÉRCIO DE SEMENTES E MUDAS.

Pesquisa de mercado. Disponível em: http:/ www.abcsem.com.br/html. Acessado em 18 de setembro de 2007.

BARBEDO CJ; NAKAGAWA J; BARBEDO ASC; ZANIN ACW. 1994. Influência da idade e do período de repouso pós-colheita de frutos de pepino cv. Rubi na qualidade fisiológica de sementes. Horticultura Brasileira 12: 118-124.

BINO RJ; AARTASE JW; VAN DER BURG WJ. 1993. Non-destructive x-ray analysis of Arabidopsis embryo mutants. Seed Science Research 3: 167-170.

BRASIL. 2009. Ministério da Agricultura e Reforma Agraria. Regras para análise de sementes. SNDA/DNDV/CLAV. 365 p.

BURG WJ; AARTESE JW; ZWOL RA; JALINK H; BINO RJ. 1994. Predicting tomato seedling morphology by X-ray analysis of seeds. Journal of the American Society for Horticultural Science 119: 258-263.

CARVALHO MLM; OLIVEIRA LM. 2006. Raios $X$ na avaliação da qualidade de sementes. Informativo ABRATES 16: 93-99.

CARVALHO MLM; SILVA CD; OLIVEIRA LM;
SILVA DG; CALDEIRA CM. 2009. Teste de raios $\mathrm{X}$ na avaliação da qualidade de sementes de abóbora. Revista Brasileira de Sementes 31: 221-227.

GAGLIARD B; MARCOS-FILHO J. 2011. Relationship between germination and bell pepper seed structure assessed by the X-ray test. Scientia Agricola 68: 411-416.

ISTA - International Rules for Seed Testing. 1993. Seed Science and Technology 21: 363 (ssupplement).

ISTA - International Rules for Seed Testing. 2004. Seed Science and Technology 27: 333. (supplement).

MENEZES NL; CÍCERO SM; VILLELA FA. 2005. Identificação de fissuras em sementes de arroz após a secagem artificial, por meio de raios X. Ciência Rural 35: 1194-1196.

NASCIMENTO WM; LIMA LB; ALVARES MC. 2000. Maturação de sementes híbridas de berinjela. Horticultura Brasileira 18: 10401041. Suplemento.

OLIVEIRA LM; CARVALHO MLM; DAVIDE AC. 2003. Utilização do teste de raios X na avaliação da qualidade de sementes de canafístula (Peltophorum dubium). Revista Brasileira de Sementes 25: 116-120.

PEDROSA JF; OLIVEIRA GM; NETO FB; MONTEIRO MR. 1987. Influência da idade e armazenamento do fruto na produção e qualidade de sementes de Cucurbita máxima x moschata. Horticultura Brasileira 5: 15-17.

PUPIM TL; NOVEMBRE ADLC; CARVALHO MLM; CICERO SM. 2008. Adequação do teste de raios $X$ para avaliação da qualidade de sementes de embaúba (Cecropia pachystachya). Revista Brasileira de Sementes 30: 28-32.

SIMAK M. 1991. Testing of forest tree and shrub seeds by x radiography. In: Gordon AG; GOSLING PG; WANG B. Tree and shrub seed handbook. International Seed Testing Association, p-14-28.

SWAMINATHAN MS; KAMRA SK. 1961. X-ray analysis of the anatomy and viability of seed of some economic plants. Indian Journal of Genetics \& Plant Breeding 4: 129-135.

TALAMINI V; RAMOS SRR. Agron Agronegócios online. Disponível em http//www.agron.com. $\mathrm{br} / \mathrm{v} / 30135$-a-escolha-da-semente-e-muitoimportante/html. Acessado em abril de 2011. 